\title{
PNA Microprobe for Label-Free Detection of Nucleic Acid Repeat Mutations
}

Received 00th January 20xx, Accepted 00th January 20xx

DOI: $10.1039 / \times 0 \times x 00000 x$

\author{
Narges Asefifeyzabadi, ${ }^{1}$ Grace Durocher, ${ }^{1}$ Kizito-Tshitoko Tshilenge,${ }^{2}$ Lisa M. \\ Ellerby, ${ }^{2}$ Mohtashim H. Shamsi ${ }^{1 *}$
}

We present a PNA-based microprobe sensing platform to detect nucleic acid repeat mutations by electrochemical impedance spectroscopy. The microprobe platform discriminated Huntington's disease-associated CAG repeats in cell-derived total RNA. This sensitive, label-free, and PCR-free detection strategy has the potential to detect a plethora of length mutation disorders.

Nucleic acid repeats of various types are responsible for more than 40 genetically transferrable diseases. ${ }^{1}$ Their detection has been challenging owing to their length. ${ }^{2}$ Current state-of-the-art detection of repeats relies on PCR-based methods and prone to frequent falsepositive and false-negative results. ${ }^{3}$ Despite the high sensitivity of the electrochemical techniques, down to attomolar level, ${ }^{4,5}$ there were a few efforts to detect expanded repeats and most of them relied on chemical labelling, detecting short target lengths (maximum 10 repeats), while standing at proof-of-concept level. ${ }^{6-11}$ DNA probes were previously proposed for label-free detection of $\mathrm{G}_{4} \mathrm{C}_{2}$ repeats in cell-derived RNA by measuring the charge transfer resistance $\left(R_{c t}\right)$ of the interface. ${ }^{12}$ Despite the label-free strategy, the sensitivity of the system was lower because difference in the detection signal between abnormal and normal target was only factor of 1.2 and the amount of RNA sample used $(1 \mu \mathrm{g} / \mu \mathrm{L})$ was relatively high for electrochemical platform. ${ }^{12}$ Lower sensitivity of surface-bound probes in electrochemical biosensing may be a challenge due to limited control of probe orientation and density, and low hybridization efficiency on surface. ${ }^{13,14}$ Peptide nucleic acid (PNA) probes can mitigate these problems due to the excellent stability and improved hybridization efficiency owing to their electrostatic neutrality and stiff backbone. 15-19

Here, we have developed a platform comprising PNA microprobes immobilized on gold surface for label-free detection of

\footnotetext{
${ }^{1}$ School of Chemical and Biomolecular Sciences, 1245 Lincoln Dr, Southern Illinois University at Carbondale, IL 62901, USA. *Email: mshamsi@siu.edu

${ }^{2}$ The Buck Institute for Research on Aging, 8001 Redwood Blvd, Novato, CA 94945, USA
}

Electronic Supplementary Information (ESI) available: [details of any supplementary information available should be included here]. See DOI: 10.1039/x0xx00000x

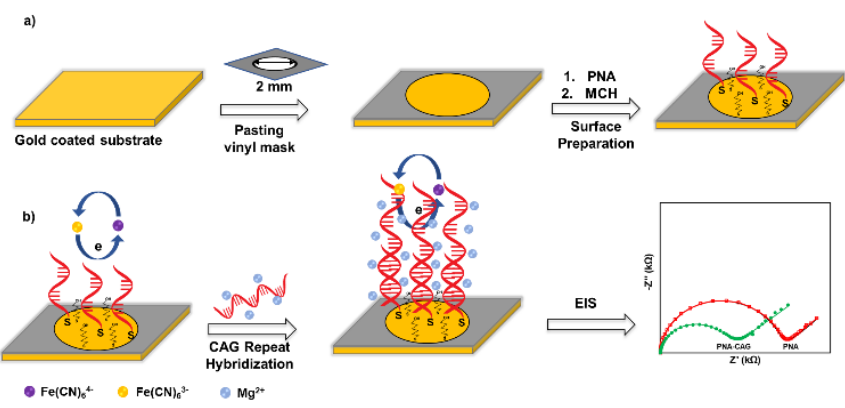

Scheme 1. a) Preparation of sensing platform by immobilizing PNA (CTG-6) probe and MCH on $2 \mathrm{~mm}$ diameter gold coated surface using vinyl sheet as a mask to expose detection area. b) Surface hybridization in presence of $\mathrm{Mg}^{2+}$ followed by EIS measurement using 1:1 $\mathrm{Fe}(\mathrm{CN})_{6}^{3-4-}$-redox probe.

trinucleotide CAG repeats associated with Huntington's disease $(H D)^{20}$ by measuring the charge transfer resistance of the biosensing platform. The PNA probe (CTG-6) was immobilized on gold-coated substrate through Au-S bond followed by immobilizing mercaptahexanol (MCH) filler layer (Scheme 1a). Then, target CAG repeats were hybridized on surface in presence of high cationic strength (20 $\mathrm{mM} \mathrm{Mg}^{2+}$ ), which led to decease in $R_{\mathrm{ct}}$ following the hybridization event (Scheme 1b). We have previously shown that addition of metal ion (e.g. $\mathrm{Zn}^{2+}$ ) into prehybridized DNA monolayers lowers the $R_{c t}$ signal, which amplifies the sequence-dependent structural deformation of the DNA at molecular level (e.g. single basepair mismatch). ${ }^{21} \mathrm{Mg}^{2+}$ was preferred in this study due to its role in stabilizing DNA duplex structure, ${ }^{22}$ which can also facilitate diffusion of the redox probe across the duplex film and exchange of electron with the electrode.

For the PNA-based electrochemical detection, gold electrodes ( 2 $\mathrm{mm}$ dia.) were prepared by cutting $1 \mathrm{~cm}^{2}$ pieces from a $100 \mathrm{~nm}$ gold coated substrate (Platypus, USA). The small substrates were immersed in an acid piranha $\mathrm{H}_{2} \mathrm{SO}_{4}: \mathrm{H}_{2} \mathrm{O}_{2}$ (3:1) for 10 second followed by washing with DI water, $100 \mu \mathrm{M}$ Tris buffer, and drying with $\mathrm{N}_{2}$. Then, a vinyl sheet with a $2 \mathrm{~mm}$ diameter hole was pasted on top of the $1 \mathrm{~cm}^{2}$ gold substrate to define the sensing electrode area. Then, a $5 \mu \mathrm{L}$ aliquot of thiol modified PNA probe 'CTG-6' (Table S1) solution was dropped on to the electrode surface to form a surfaceassembled film on the surface at $4{ }^{\circ} \mathrm{C}$. The electrodes were kept in a 
humid closed container to prevent evaporation. Then, the modified electrode was washed with the Tris buffer and incubated with $1 \mathrm{mM}$ mercaptahexanol (MCH) for 30 minutes to block any unmodified surface around the probe. The PNA-MCH modified electrode was later washed again with the buffer and dried with $\mathrm{N}_{2}$ before exposing to CAG repeat targets. Table $\mathbf{S 1}$ in the supplementary information shows the details of the repeat sequences that were used for this study. PNA probe CTG-6 (PNA Bio, USA) was thiol modified at 5 '-end while the synthetic DNA targets (IDT, USA) were CAG repeats with 6 , 10,15 , and 20 repeat lengths. DNA and RNA sample solutions were prepared in $100 \mu \mathrm{M}$ Tris containing $20 \mathrm{mM} \mathrm{MgCl}_{2}$ and $200 \mathrm{mM} \mathrm{NaCl}$ $(\mathrm{pH}=8.5)$. Total RNA containing 72CAG $(\mathrm{HD}+)$ and 21CAG (HD-) were isolated from neural stem cells as described previously. ${ }^{23}$ Extraction details of the RNA samples are given in the supplementary information. RNA samples were diluted to $10 \mathrm{ng} \cdot \mu \mathrm{L}^{-1}$ concentration for electrochemical detection. All electrochemical experiments were performed using Autolab (Metrohm, USA) at room temperature in a three-electrode cell using the modified gold electrode as working electrode, Pt wire counter electrode, and $\mathrm{Ag} / \mathrm{AgCl}$ reference electrode. Electrochemical impedance spectroscopy (EIS) was performed using soluble redox probe, $1 \mathrm{mM} \mathrm{K}_{4}\left[\mathrm{Fe}(\mathrm{CN})_{6}\right] / \mathrm{K}_{3}\left[\mathrm{Fe}(\mathrm{CN})_{6}\right]$ (1:1) prepared in $5 \times$ PBS buffer ( $\mathrm{pH}$ 7.2). The following parameters were used to run the EIS: Frequency range $100 \mathrm{kHz}$ to $1 \mathrm{~Hz}$, an applied $\mathrm{DC}$ potential of $250 \mathrm{mV}$ vs. $\mathrm{Ag} / \mathrm{AgCl}$, and $\mathrm{AC}$ pulse of $5 \mathrm{mV}$ amplitude. For simulation, Z-view version $3.5 \mathrm{~d}$ was used to fit the EIS data into a modified Randle's equivalent circuit and extract the values for fitting elements including charge transfer resistance $\left(R_{c t}\right)$.
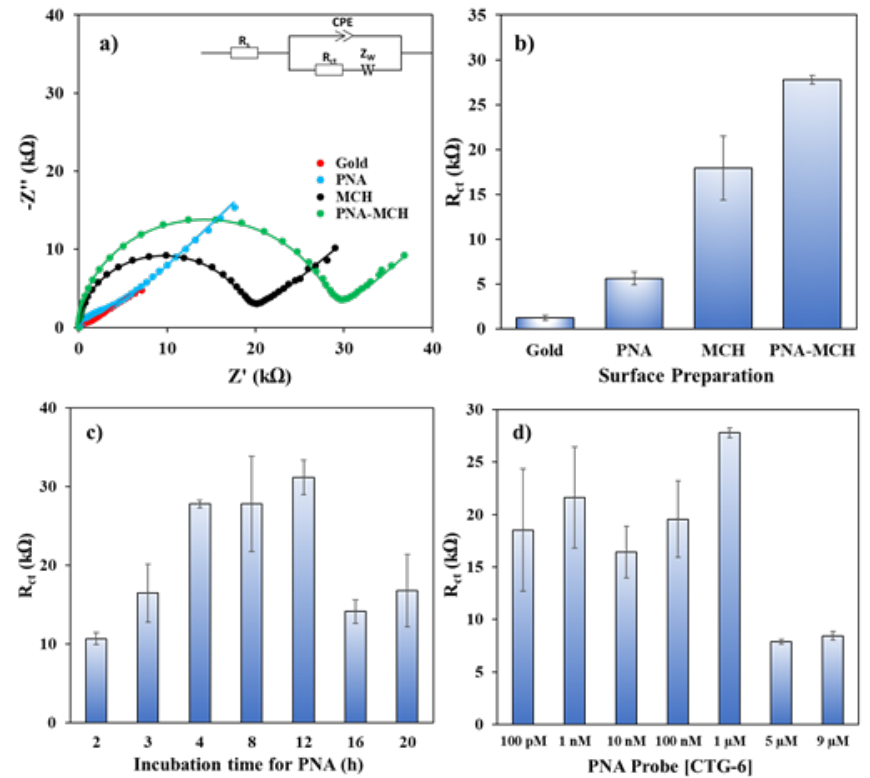

Figure 1. a) Nyquist plots and $\mathbf{b}$ ) Bar graph of bare gold electrode, PNA probe, $\mathrm{MCH}$, and PNA-MCH. The inset in (a) shows the modified Randle's equivalent circuit used for fitting the EIS measurements. c) $R_{c t}$ response of PNA-MCH versus time of incubation. d) $R_{c t}$ response of PNA-MCH versus concentration of PNA in PNA-MCH layer. The error was calculated for $\mathrm{N} \geq 3$ separate measurements.

Figure 1 shows the EIS response of various layers of the sensing platform and optimized conditions for the probe immobilization. The Nyquist form of EIS responses (Figure 1a) of bare gold, 'PNA' probe, ' $\mathrm{MCH}$ ' layer, and the combined 'PNA-MCH' layer. The inset shown in the panel is the modified Randel's equivalent circuit that fits the EIS
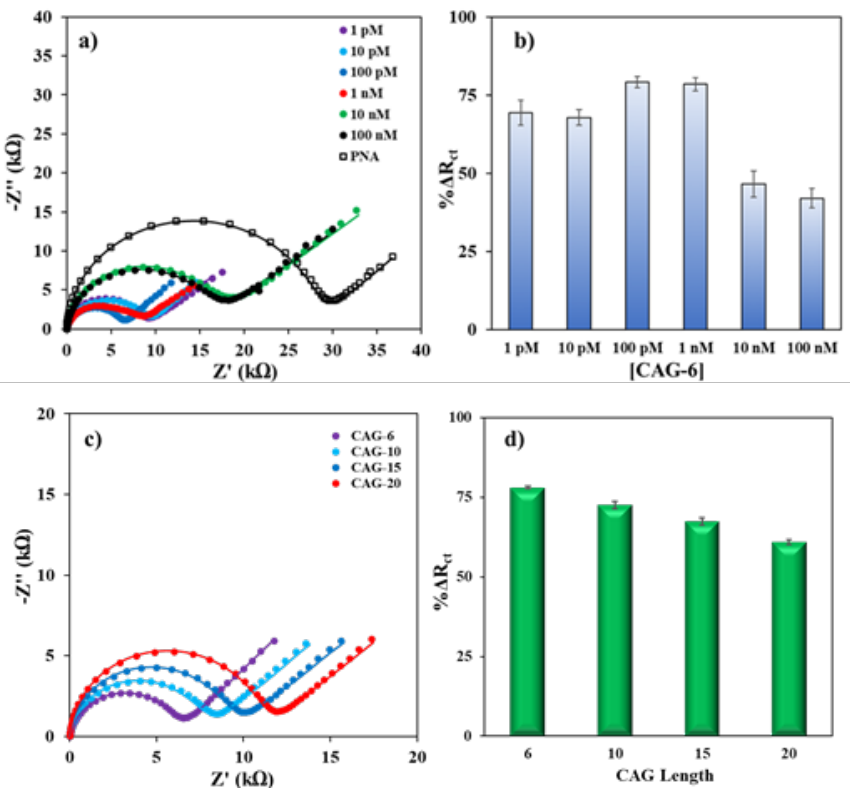

Figure 2. Detection of CAG-6 target. a) Nyquist form of EIS plots with curve fitting before and afte hybridization between the PNA probe and various concentrations of the target. b) Bar graph of the $\% \Delta R_{\text {ct }}$ values versus target concentrations. c) Nyquist form of EIS plots with curve fitting for various lengths of CAG targets. d) Bar graph of the $\% \Delta R_{\text {ct }}$ values versus $C A G$ target length where CAG Target concentration was $100 \mathrm{pM}$

data (extracted fitting values are provided in the supplementary information Table S2). The bar graph in Figure 1b shows that the probe only response (PNA) has a very low $R_{c t}$ due to the absence of negative charge, which is due to the absence of any electrostatic repulsion between the probe and the negatively charged redox probe. Thus, the resistance to charge transfer from 'PNA' is only a physical barrier while there may be a leakage current through empty space present around the probe. ${ }^{24}$ While the mercaptahexanol blocking layer ( $\mathrm{MCH}$ ) has a higher $\mathrm{R}_{\mathrm{ct}}$ than the PNA due to its higher film packing and negative charge on the surface due to $-\mathrm{OH}$ group. The response of the platform with PNA followed by $\mathrm{MCH}$ immobilization (PNA-MCH) has higher $R_{c t}$ than the separate layers with significant reproducibility where $\mathrm{MCH}$ blocks the non-specific sites and prevent current leakage through the pinholes. The $R_{c t}$ of PNA-MCH at $4 \mathrm{~h}$ immobilization time (Figure 1c) and at $1 \mu \mathrm{M}$ PNA concentration (Figure 1d) was the highest and has the lowest RSD = 1.7\% (see EIS curves in Figure S1). Based on the results in Figure 1, the PNA-MCH platform was prepared to detect the CAG repeat targets.

$$
\% \Delta \text { Rct }=\frac{\text { (Probe Rct }- \text { Target Rct })}{\text { Probe Rct }} \times 100
$$

Then, complementary target CAG- 6 ( 6 repeat units same as probe length) was hybridized at $0.25-2 \mathrm{~h}$ to optimize the hybridization time. The $R_{\mathrm{ct}}$ decreased following hybridization (Figure S2a in supplementary information) due to high cationic strength microenvironment, which reduces electrostatic repulsion between the sensing interface and the negatively charged redox probe. The $\% \Delta R_{c t}$ was calculated using Eq. 1 and the results were compared in Figure S2b. The hybridization time of $\mathbf{3 0}$ min was selected for further experiments where the change in signal reaches steady-state and has the lowest standard error, i.e. $59.0 \pm 0.7 \%$. Figure 2 shows the EIS 
response of CAG-6 detection at various concentrations. Figure 2a represents the Nyquist form of EIS curves of CAG- 6 target between 1 $\mathrm{pM}-100 \mathrm{nM}$. The $\% \Delta \mathrm{R}_{\mathrm{ct}}$ with respect to CAG-6 concentration in Figure $\mathbf{2} b$ shows the highest change in $\mathrm{R}_{\mathrm{ct}}$ following duplex formation at $100 \mathrm{pM}$ with $79.2 \pm 1.7 \%$. Figure $\mathbf{2 c}$ represents the Nyquist plots of 6-20 CAG repeats measured at 100 pM concentration, which reveals that the increase in repeat length resulted in higher $R_{c t}$ and hence lower $\% \Delta \mathrm{R}_{\mathrm{ct}}$ (Figure $\mathbf{2 d}$ ). Higher resistance to charge transfer at higher repeat length is attributed to steric hindrance caused by size of the target sequence and consequently resisting diffusion of the redox probe through the sensing interface. Nevertheless, the repeat lengths were distinguishable from each other.

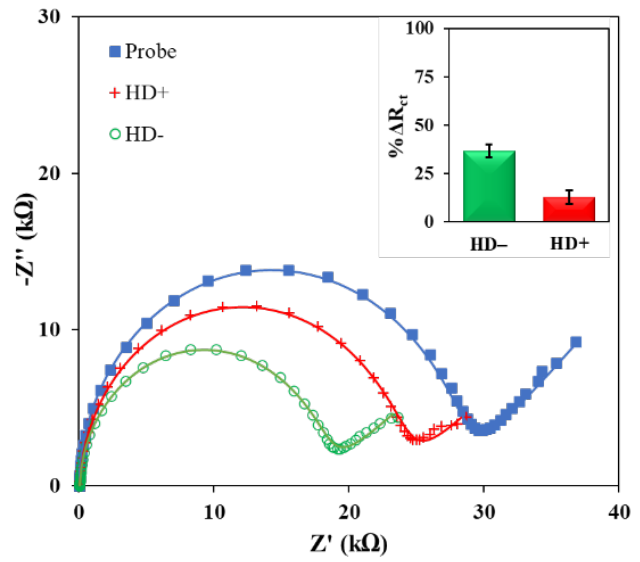

Figure 3. EIS Response of the PNA microprobe, HD- (21CAG), and HD+ (72 CAG) repeat in total RNA extracted from neural stem cells. The inset shows the $\% \Delta R_{\text {ct }}$ of the $H D-(21 C A G)$, and $H D+(72 C A G)$ repeat after hybridization. The error bar represents standard deviation for $\mathrm{N}=5$.

Finally, the PNA microprobe detection platform was applied to discriminate normal and pathogenic CAG repeats in real sample. The total RNA for the detection was extracted from neural stem cells as described in the supplementary information and $10 \mathrm{ng} \cdot \mathrm{LL}^{-1}$ concentration. CAG expands up to 35 repeats in a normal individual while pathogenicity begins when it expands beyond 38 repeats resulting in Huntington's disease (HD). ${ }^{25}$ The total RNA sample with normal length (HD-) contains 21CAG repeats while with the pathogenic length $(\mathrm{HD}+)$ carries 72CAG repeats. Figure 3 shows the $\mathrm{R}_{\mathrm{ct}}$ signals of the HD-, HD+, and PNA microprobe at two sample concentrations. In the complex sample, the overall change was lower than the purified target oligonucleotides presented above. Nevertheless, there was a significant difference between the normal and pathogenic repeats. There was a higher change in HD- $(38.0 \pm$ $3.3 \%)$ compared to $\mathrm{HD}+(12.7 \pm 3.6 \%)$. This corroborated the trend with respect to length variation obtained above (see Figure $2 \mathbf{d}$ ). There was almost 3-fold difference between the average signals of $\mathrm{HD}$ - and $\mathrm{HD}+$, which is also aligned with the length difference between the normal and abnormal repeat lengths.

Here, we have reported PNA microprobe-based label-free electrochemical platform for the detection of repeat length mutations. The detection platform was optimized for the detection of CAG repeats associated with Huntington's disease. Concentration and immobilization time for the probe were optimized to obtain best performance to detect CAG targets of various concentrations and lengths. The conditions with the lowest error were selected to discriminate normal and abnormal CAG repeats in neural cellextracted total RNA. The sensitivity of the PNA microprobe is significantly better than DNA probe reported previously for $\mathrm{G}_{4} \mathrm{C}_{2}$ repeats, ${ }^{12}$ by achieving 3:1 HD- signal/HD+ signal at $10 \mathrm{ng} \cdot \mu^{-1}$, which is 2.5-fold signal improvement in 100 -fold lower sample concentration. To best of our knowledge, this is the first application of PNA microprobe to discriminate length mutations by simple $R_{c t}$ measurement. In future, sensitivity of the PNA microprobes and the label-free electrochemical detection mechanism will transform the diagnosis of the diseases owing to length mutation into low-cost platforms and less susceptible to false-positive/false-negative results. $^{5}$

\section{Conflict of Interest}

There are no conflicts to declare.

\section{Acknowledgement}

MHS acknowledges National Science Foundation EAGER award CBET-1940716 to support this research.

\section{References}

1. K. J. Rohilla and K. T. Gagnon, Acta Neuropathol Commun, 2017, 5, 63 .

2. S. M. Mirkin, Curr Opin Struc Biol, 2006, 16, 351-358.

3. A. Crook, A. McEwen, J. A. Fifita, K. Zhang, J. B. Kwok, G. Halliday, I. P. Blair and D. B. Rowe, Amyotrophic lateral sclerosis \& frontotemporal degeneration, 2019, 20, 310-316.

4. Y. Sun, X. He, J. Ji, M. Jia, Z. Wang and X. Sun, Talanta, 2015, 141, 300-306.

5. N. Asefifeyzabadi, R. Alkhaldi, A. Z. Qamar, A. A. Pater, M. Patwardhan, K. T. Gagnon, S. Talapatra and M. H. Shamsi, ACS Applied Materials \& Interfaces, 2020, 12, 52156-52165.

6. Y. L. Liu, J. Li, G. Chang, R. Z. Zhu, H. P. He, X. H. Zhang and S. F. Wang, New J Chem, 2018, 42, 9757-9763.

7. X. Q. Zhu, J. Li, H. H. Lv, H. P. He, H. Liu, X. H. Zhang and S. F. Wang, Rsc Adv, 2017, 7, 36124-36131.

8. J. Li, Y. Liu, X. Zhu, G. Chang, H. He, X. Zhang and S. Wang, ACS applied materials \& interfaces, 2017, 9, 44231-44240.

9. M. Fojta, L. Havran, M. Vojtiskova and E. Palecek, J Am Chem Soc, 2004, 126, 6532-6533.

10. F. Miroslav, B. Petra, C. Kateřina and P. Petr, Electroanalysis, 2006, 18, 141-151.

11. I. V. Yang and H. H. Thorp, Anal Chem, 2001, 73, 5316-5322.

12. M. Taki, K. J. Rohilla, M. Barton, M. Funneman, N. Benzabeh, S. Naphade, L. M. Ellerby, K. T. Gagnon and M. H. Shamsi, Analytical and Bioanalytical Chemistry, 2019, 411, 6995-7003.

13. F. Lucarelli, G. Marrazza, A. P. F. Turner and M. Mascini, Biosensors and Bioelectronics, 2004, 19, 515-530.

14. J. I. A. Rashid and N. A. Yusof, Sensing and Bio-Sensing Research, 2017, 16, 19-31. 
15. C. Briones and M. Moreno, Anal Bioanal Chem, 2012, 402, 3071-3089.

16. S. Barluenga and N. Winssinger, Accounts of Chemical Research, 2015, 48, 1319-1331.

17. J. B. Raoof, R. Ojani, S. M. Golabi, E. Hamidi-Asl and M. S. Hejazi, Sensors and Actuators B: Chemical, 2011, 157, 195-201.

18. M. S. Hejazi, M. H. Pournaghi-Azar, E. Alipour, E. D. Abdolahinia, S. Arami and H. Navvah, Electroanal, 2011, 23, 503-511.

19. K. Bartold, A. Pietrzyk-Le, K. Golebiewska, W. Lisowski, S. Cauteruccio, E. Licandro, F. D'Souza and W. Kutner, Acs Appl Mater Inter, 2018, 10, 27562-27569.

20. L. M. Ellerby, Neurotherapeutics, 2019, 16, 924-927.

21. M. H. Shamsi and H.-B. Kraatz, Analyst, 2010, 135, 2280-2285.

22. M. H. Shamsi and H.-B. Kraatz, Journal of Inorganic and Organometallic Polymers and Materials, 2013, 23, 4-23.

23. A. Z. Qamar, N. Asefifeyzabadi, M. Taki, S. Naphade, L. M. Ellerby and M. H. Shamsi, Journal of Materials Chemistry $B$, 2020, 8, 743-751.

24. M. H. Shamsi and H.-B. Kraatz, Analyst, 2011, 136, 3107-3112.

25. N. Zhang, B. J. Bailus, K. L. Ring and L. M. Ellerby, Brain Res, 2016, 1638, 42-56. 